\title{
The Diagnostic Value of Current Practice of Pediatric Intravenous Urography in Afghanistan in Presence of Ultrasonography; Cross-Sectional Analytic Study at FMIC, Kabul
}

\author{
Hidayatullah Hamidi, ${ }^{1, *}$ and Najibullah Rasouly ${ }^{1}$ \\ ${ }^{1}$ French Medical Institute for Children (FMIC), Kabul, Afghanistan \\ "Corresponding author: Hidayatullah Hamidi. E-mail: hedayatullah.hamidi@gmail.com
}

Received 2016 December 21; Accepted 2017 February 08.

\begin{abstract}
Background: Intravenous urography (IVU); the oldest imaging method for assessing the upper urinary tract anatomy and function; is now seldom used in the pediatric population. The important concern associated with IVU is the radiation exposure. Due to various reasons, IVU is still widely practiced in pediatrics in Afghanistan. On the other hand, Ultrasonography (USG) is universally accepted as the first-line imaging modality in pediatric uroradiology. It is inexpensive, immediate, painless, radiation-free, non-invasive and widely available.

Objectives: To assess the diagnostic value of current practice of pediatric IUV in presence of USG in Afghanistan. To compare the validity OF USG, plain abdominal radiograph, and their combination in with IVU in detection of urolithiasis in children.

Methods: A prospective cross-sectional analytic study was conducted on pediatric patients at radiology department of FMIC. First USG of urinary tract was done for all participants, followed by IVU. The statistical analysis was done using SPSS version 23 . The diagnostic value of IVU was considered positive when it could provide additional information over USG and negative if it could not give any addition information over USG. Accuracy of USG, plain radiograph and their combination in detection of urolithiasis was calculated taking IVU as reference examination.

Results: The diagnostic value of IVU was found positive in $6.5 \%$ while it was negative in (93.5\%) cases. The sensitivity and specificity of USG, Rad and their combination in detection of urolithiasis was calculated as $90.5 \%$ and $84.8 \%, 84.1 \%$ and $88.6 \%$; and $98.4 \%$ and $4.8 \%$ respectively.

Conclusions: IVU can be largely replaced by USG in the pediatric population and if really clinically needed, its indications should always be judged according to clinical context and USG findings. In cases of urolithiasis combination of USG and plain radiograph can mostly answer the clinical question and there may be no need to perform IVU.
\end{abstract}

Keywords: Intravenous Urography, Pediatric Uroradiology, Ultrasonography

This is an abstract presented in the 33rd Iranian congress of radiology (ICR) and the 15th congress of Iranian radiographic science association (IRSA). 